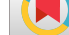

\section{Association Between Tumor Necrosis Factor- $\alpha$ and the Risk of Hepatic Events: A Median 3 Years Follow-Up Study}

\author{
Zhi Zhu, ${ }^{1}$ and Shuofeng $\mathrm{Li}^{1}{ }^{1,}$ \\ ${ }^{1}$ Department of Radiology, Cangzhou Central Hospital, Cangzhou, Hebei Province, China \\ "Corresponding author: Shuofeng Li, Department of Radiology, Cangzhou Central Hospital, Cangzhou, Hebei Province, China. E-mail: lsfo4221025@126.com
}

Received 2017 December 25; Revised 2018 March 11; Accepted 2018 May 18.

\begin{abstract}
Background: Tumor necrosis factor- $\alpha$ (TNF- $\alpha$ ) is thought to be involved in the development of non-alcoholic fatty liver disease (NAFLD). However, it is still undetermined whether TNF- $\alpha$ is a significant indicator of major adverse hepatic events (MAHE).

Methods: A total of 279 participants with TNF- $\alpha$ data were enrolled and assigned into three different groups based on the serum TNF- $\alpha$ level. The severity of NAFLD was evaluated with magnetic resonance imaging to determine liver proton density fat fraction. The association between TNF- $\alpha$ and MAHE as well as the association between TNF- $\alpha$ and all-cause death were evaluated with the risk-djusted Cox Proportional-hazards Model.

Results: Compared with those in the lowest tertile, participants with highest TNF- $\alpha$ level displayed higher serious hepatic events or burden. After a median 3 years follow-up period, participants with highest TNF- $\alpha$ had a higher risk of MAHE (HR 1.74, 95\% CI 1.05 $1.93, \mathrm{P}<0.001)$ and all-cause death rates (HR 2.63, 95\% CI 1.89-3.35, P<0.001) than those with low TNF- $\alpha$ level subjects.

Conclusions: Patients with high level of TNF- $\alpha$ have increased the burden of NAFLD and higher MAHE risk compared to patients with low level of TNF- $\alpha$.
\end{abstract}

Keywords: Tumor Necrosis Factor- $\alpha$, Non-Alcoholic Fatty Liver Disease, Magnetic Resonance Imaging, Inflammation

\section{Background}

Hepatic events, especially non-alcoholic fatty liver disease (NAFLD), which affects $20 \%-40 \%$ of the adult population ranging from hepatic steatosis to non-alcoholic steatohepatitis (NASH), are generally considered asymptomatic and notoriously serious until decompensation occurs (1-3). Decompensation mostly occurs in the presence of steatosis and inflammation. Therefore, when assessing the risk of hepatic events, it is important to give insight into prognosis of advanced steatosis and inflammation (4$6)$.

The exact nature of NAFLD is still unknown. However, certain conditions such as obesity, insulin resistance, and inflammation probably play a strong role in the disease progress. Assessment of the severity of steatosis is important in the evaluation of the stage of NAFLD, due to the fact that the prevalence of steatosis associated with obesity is dramatically affecting developed countries $(1,6)$.

Although liver biopsy is traditionally recommended as the gold standard to diagnose and grade steatosis, inflammation, and fibrosis, the poor patient acceptance and the risk of non-representative sampling error further restrict the widespread application $(7,8)$. In recent years, the development and application of non-invasive tests of NAFLD became a hot research area and have revolutionized the hepatology practice. Numerous studies have confirmed the accuracy of non-invasive tests in steatosis, steatohepatitis staging, and diagnosis (8-11). Among them, serum assay and magnetic resonance imaging (MRI) evaluation have become valuable tools to non-invasively diagnose and monitor hepatic events.

Obesity and inflammation are closely linked with NAFLD. It is of clinical interest to assess the liver fat fraction and inflammation molecules as a hallmark of NAFLD development and progression. MRI determined that liver proton density fat fraction not only correlates with histologydetermined steatosis grade in adults with NAFLD, however, it also accurately quantifies hepatic steatosis in NAFLD, which can be used to monitor disease progression and is considered a potential alternative to liver biopsy in clinical practice $(7,12)$. Moreover, as one of the most important adipocytokines, tumor necrosis factor alpha (TNF- $\alpha$ ) and its receptor is thought to be involved in the development of NAFLD and sustains chronic inflammation leading to liver fibrosis $(13,14)$. TNF- $\alpha$ secretion is induced by a vari-

Copyright (c) 2018, Hepatitis Monthly. This is an open-access article distributed under the terms of the Creative Commons Attribution-NonCommercial 4.0 International License (http://creativecommons.org/licenses/by-nc/4.0/) which permits copy and redistribute the material just in noncommercial usages, provided the original work is properly cited. 
ety of stimuli, including fat accumulation, and organ damage. If the stimulus persists, the inflammation can become chronic. However, it is still undetermined whether TNF- $\alpha$ is a significant indicator of major adverse hepatic events (MAPE), including hepatic decompensation, hepatocarcinoma, and liver-related death for participants without the history of NAFLD who underwent MRI assessment of liver fat fraction.

In this assay, we investigated the potential role of serum TNF- $\alpha$ level as a useful clinical biomarker to predict the risk of future hepatic events in Chinese participants without NAFLD history who underwent an MRI.

\section{Methods}

\subsection{Study Population}

A total of 325 participants without a history of NAFLD undergoing MRI evaluation were retrospectively enrolled and only those participants (279 participants) with available serum TNF- $\alpha$ level data were included in the present study. The study procedure was approved by the ethics committee of Cangzhou Central hospital, and written informed consent forms were given by all participants. Then, the participants were assigned into three different groups based on serum TNF- $\alpha$ levels $(\leq 1.60,1.61-3.10$, and $\geq 3.11$ $\mathrm{pg} / \mathrm{mL})$.

The clinical diagnosis was made using ICD-9 codes from inpatient and outpatient encounters, such as 570 (acute or subacute liver necrosis), 571.5 (cirrhosis without the use of alcohol), 572.2 (hepatic coma), and 573.3 (toxic, noninfectious hepatitis). These events of NAFLD were considered serious if the diagnosis was recorded as part of a hospitalization; all others were considered non-serious NAFLD (15).

\subsection{Procedures}

Demographic data and hepatic events risk factors, such as gender, age, family history, smoking status, diabetes, dyslipidemia, body mass index (BMI), waist circumference, low-density lipoprotein (LDL), high-density lipoprotein (HDL), glucose, liver fat fraction, liver volume, visceral fat volume, and subcutaneous fat volume were acquired by a standardized questionnaire and clinical assay. Smoking status was classified into never, past, or current smoker (at least 6 months, at least one cigarette per day) (16). Diabetes was defined as hemoglobin A1c (HbA1c) higher than $6.5 \%$. Body mass index (BMI) was computed as body weight/ (height ${ }^{2}$ ). Waist circumferences (narrowest diameter between the xiphoid process and iliac crest) were measured. TNF- $\alpha$ was measured by ELISA (BD Biosciences, Franklin Lakes, NJ, USA), according to the manufacturer's instructions.

\subsection{MRI for Liver Fat Fraction Quantification}

Baseline and follow-up MRI were used to assess changes in liver fat fraction utilizing a most advanced MRIdetermined proton density fat fraction (MRI-PDFF) method (12). A standard non-contrast enhanced T1-weighted VIBE (volume interpolated breath-hold examination) sequence combined with a Dixon fat separation technique (spatial resolution $2.9 \times 2.0 \times 3.0 \mathrm{~mm}^{3}$; acquisition time $28 \mathrm{~s}$; and echo times $2.39 \mathrm{~ms}$ and $4.78 \mathrm{~ms}$ ) was adopted and performed with 1.5 T MRI system (Verio, Siemens, Erlangen, Germany) (17). OsiriX 4.0 (OsiriX Foundation, Geneva, Switzerland) was used to analyze the images. Three consecutive slices were scanned in the right liver lobe and two consecutive slices were scanned in the left lobe. The center slice was at the level of the portal vein. The second level of measurement for both lobes was two consecutive slices above the center and additionally on the right side two slices below the center. The MRI-PDFF was calculated using the following previously described equation: $\mathrm{FF}=$ fat $/$ (fat + water).

All scans were independently assessed by two radiologists blind to the group assignment and clinical information and a final decision was obtained based on consensus interpretation.

\subsection{Endpoints}

The endpoints of this study were all-cause death and MAHE, which included fibrosis or cirrhosis, liver failure, and hepatocellular carcinoma.

\subsection{Statistical Analysis}

Wilcoxon Rank-sum Test was used to analyze intermgroup comparisons across tertiles. Risk-adjusted and unadjusted Cox Proportional-hazards Model were employed to examine the relationship between TNF- $\alpha$ level and MAHE or all-cause death. The hazard ratio (HR) and 95\% confidence interval (CI) were determined by the Cox Model. $\mathrm{P}<0.05$ was considered to be statistically significant. Free survival curves of MAHE and all-cause-death were generated across different tertiles using the multivariate Cox Proportional-hazards Model. GraphPad Prism 6.00 (GraphPad Software, San Diego, CA) was used to draw the figures.

\section{Results}

\subsection{Baseline Characteristics}

Among 325 participants without known NAFLD undergoing MRI assessment, TNF- $\alpha$ detection was available for 279 participants (85.8\%; Figure 1). The baseline demographic and clinical characteristics of participants based 


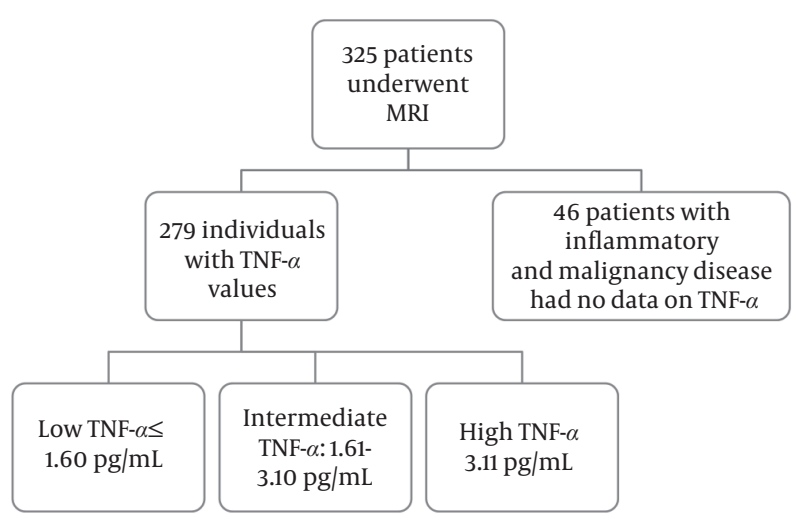

Figure 1. Study flow chart

on TNF- $\alpha$ tertiles were listed in Table 1 . Participants with high TNF- $\alpha$ serum level displayed a higher rate of BMI (P $=0.0362)$, LDL $(P=0.0293)$, and glucose levels $(P=0.0396)$ compared with participants in the low TNF- $\alpha$ group. Furthermore, participants with high TNF- $\alpha$ level exhibited a significantly elevated rate of diabetes $(\mathrm{P}=0.0007)$, dyslipidemia $(P=0.0004)$, and waist circumference $(P=0.0072)$. However, liver fat fraction $(\mathrm{P}=0.0505)$, liver volume $(\mathrm{P}=$ $0.0868)$, visceral fat volume $(\mathrm{P}=0.0507)$, and subcutaneous fat volume $(\mathrm{P}=0.0753)$ did not show significant differences among different groups in the initial phase of observation.

\subsection{Severity of NAFLD}

The severity of NAFLD evaluated by normal, nonserious, and serious hepatic events is showed in Table 2. Compared with participants with low TNF- $\alpha$ level, those with high TNF- $\alpha$ displayed a greater rate of serious hepatic events, while there was no significant difference at the rate of non-serious hepatic events $(\mathrm{P}=0.0003)$. Compared with participants in lower TNF- $\alpha$ group, those with higher serum TNF- $\alpha$ showed increased liver fat fraction value $(\mathrm{P}=$ $0.0008)$. Such phenomenon can also be seen after 3 years follow-up study (Figure 2).

\subsection{Rates and Adjusted Risk of MAHE and All-Cause Death}

After $3.0 \pm 2.6$ years of follow-up study, MAHE ( $\mathrm{P}=$ $0.0009)$ and all-cause death rates $(P=0.0002)$ were found to be progressively increased with elevating TNF- $\alpha$ levels using the unadjusted Cox Model. Using the adjusted Cox Proportional-hazards Model, adjusted for gender, age, diabetes, dyslipidemia, family history, current smoke, BMI, waist circumference, glucose, and liver fat fraction, participants with highest TNF- $\alpha$ had higher risk of MAHE (HR $1.74,95 \% \mathrm{CI} 1.05-1.93, \mathrm{P}<0.001)$ and all-cause death rates (HR 2.63, 95\% CI 1.89 - 3.35, P < 0.001) than those with low

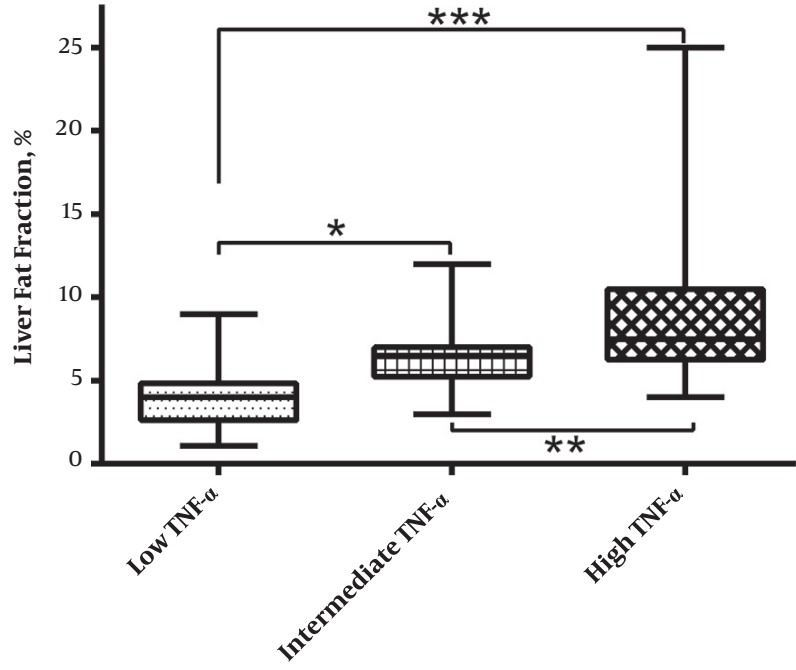

Figure 2. Box plots of liver fat fraction among three different groups based on serum TNF- $\alpha$ level. ${ }^{*} \mathrm{P}<0.05,{ }^{* *} \mathrm{P}<0.01$, and ${ }^{* * *} \mathrm{P}<0.001$

TNF- $\alpha$ level subjects (Table 3). Free survival curves also clearly showed increased MAHE and all-cause death in participants with a higher level of TNF- $\alpha$ (Figure 3).

\section{Discussion}

As the most common form of the chronic liver disease in obese patients, most NAFLD is asymptomatic and the diagnosis often followed by abnormal findings on routine examination, or abdominal MRI, was adopted for other examination related with hepatic risk events. While with a prevalence of approximately $20 \%-30 \%$ in adult population, NAFLD might be a progressive disease: over a 10-year period, up to $20 \%$ of patients with NASH will develop cirrhosis, and 10\% will suffer death related disease (18). However, due to lack of effective therapies for NAFLD and the non-acceptance of liver biopsy, the identification of risk factors for MAHE would help to give a higher insight into prognosis (8).

Obesity, insulin resistance, and inflammation are considered to be related with the development of $\operatorname{NAFLD~}(5,6$, 19). While due to the relatively short duration and possibly mild degree of the underlying obesity in Chinese patients with NAFLD, the histological severity of steatohepatitis and fibrosis are generally mild. Some evidence has linked TNF- $\alpha$ with the metabolic abnormalities of insulin resistance. Adipose tissue has been approved to be a site for TNF- $\alpha$ synthesis and increased TNF- $\alpha$ levels favor the development of insulin resistance and impaired glucose tolerance. Moreover, TNF- $\alpha$ has a central role in the develop- 


\begin{tabular}{|c|c|c|c|c|c|}
\hline & Total $(\mathrm{N}=279)$ & Low TNF- $\alpha(\mathbf{N}=\mathbf{9 3})$ & Intermediate TNF- $\alpha(\mathbf{N}=\mathbf{9 3})$ & High TNF- $\alpha(\mathbf{N}=93)$ & PValue \\
\hline TNF- $\alpha, \mathbf{p g} / \mathbf{m L}$ & & $\leq 1.60$ & $1.61-3.10$ & $\geq 3.11$ & 0.0005 \\
\hline Male gender & $152(54.5)$ & $51(54.83)$ & $51(54.83)$ & $50(53.76)$ & 0.2584 \\
\hline Age, $y$ & $52.5 \pm 10.26$ & $51.2 \pm 9.12$ & $52.3 \pm 9.85$ & $52.7 \pm 10.02$ & 0.1735 \\
\hline Family history & $87(31.18)$ & $28(30)$ & $29(32)$ & $30(32.3)$ & 0.0748 \\
\hline Current smoke & $85(30.46)$ & $22(23.66)$ & $28(30.1)$ & $35(37.63)$ & 0.0847 \\
\hline Diabetes & $50(17.9)$ & $13(13.98)$ & $16(17.2)$ & $21(22.58)$ & 0.0007 \\
\hline Dyslipidemia & $189(67.7)$ & $57(61.29)$ & $61(65.59)$ & $71(76.34)$ & 0.0004 \\
\hline BMI, $\mathbf{k g} / \mathrm{m}^{2}$ & $26.34 \pm 1.13$ & $26.57 \pm 1.12$ & $26.78 \pm 1.21$ & $27.89 \pm 1.13$ & 0.0362 \\
\hline Waist circumference, $\mathrm{cm}$ & $97.3 \pm 8.5$ & $94.3 \pm 7.6$ & $96.5 \pm 9.7$ & $99.6 \pm 8.9$ & 0.0072 \\
\hline LDL, $\mathbf{m m o l} / \mathbf{L}$ & $3.2 \pm 0.9$ & $2.8 \pm 1.1$ & $3.1 \pm 0.9$ & $3.4 \pm 0.8$ & 0.0293 \\
\hline HDL, mmol/L & $1.1 \pm 0.4$ & $1.1 \pm 0.4$ & $1.2 \pm 0.5$ & $1.2 \pm 0.3$ & 0.1623 \\
\hline Glucose, $\mathrm{mmol} / \mathrm{L}$ & $5.8 \pm 1.5$ & $5.6 \pm 1.4$ & $6.1 \pm 1.3$ & $6.2 \pm 1.5$ & 0.0396 \\
\hline Liver fat fraction, $\%$ & $2.5 \pm 1.2$ & $2.2 \pm 1.5$ & $2.4 \pm 1.7$ & $2.3 \pm 1.1$ & 0.0305 \\
\hline Liver volume, $\mathrm{cm}^{3}$ & $1946 \pm 686$ & $1940 \pm 510$ & $1944 \pm 398$ & $1949 \pm(450$ & 0.0868 \\
\hline Visceral fat volume, $\mathrm{cm}^{3}$ & $612 \pm 457$ & $605 \pm 446$ & $614 \pm 420$ & $617 \pm 465$ & 0.0507 \\
\hline Subcutaneous fat volume, $\mathrm{cm}^{3}$ & $2616 \pm 786$ & $2645 \pm 748$ & $2601 \pm 698$ & $2633 \pm 753$ & 0.0753 \\
\hline
\end{tabular}

Abbreviations: BMI, Body Mass Index; HDL, High-Density Lipoprotein; LDL, Low-Density Lipoprotein; TNF- $\alpha$, Tumor Necrosis Factor- $\alpha$.

${ }^{a}$ Values are expressed as No. (\%) or mean \pm SD.

Table 2. The Extent and Severity of Non-Alcoholic Fatty Liver Disease (NAFLD) ${ }^{a}$

\begin{tabular}{|c|c|c|c|c|c|}
\hline & Total $(\mathrm{N}=\mathbf{2 7 9})$ & Low TNF- $\alpha(\mathbf{N}=\mathbf{9 3})$ & Intermediate TNF- $\alpha(\mathbf{N}=\mathbf{9 3})$ & High TNF- $\alpha(\mathbf{N}=93)$ & PValue \\
\hline Normal & $117(41.9)$ & $51(54.84)$ & $40(43)$ & $26(27.96)$ & 0.0003 \\
\hline Non-obstructive disease & $103(36.9)$ & $34(36.56)$ & $36(38.72)$ & $37(36.56)$ & 0.0079 \\
\hline Obstructive disease & $59(21.2)$ & $8(8.6)$ & $17(18.28)$ & $30(32.26)$ & 0.0006 \\
\hline LFF & 6.626 .5 & $3.92 \pm 4.7$ & $6.56 \pm 4.3$ & $9.38 \pm 3.4$ & 0.0008 \\
\hline $\mathbf{L F F}<6$ & $117(41.94)$ & $40(43)$ & $39(41.94)$ & $37(39.78)$ & 0.0743 \\
\hline LFF 6 - 9 & $112(40.14)$ & $32(34.4)$ & $37(39.78)$ & $43(46.24)$ & 0.0082 \\
\hline LFF $>\mathbf{9}$ & $50(17.92)$ & $10(10.75)$ & $17(18.28)$ & $23(24.73)$ & 0.0005 \\
\hline
\end{tabular}

Abbreviation: LFF, Liver Fat Fraction.

${ }^{\mathrm{a}}$ Values are expressed as No. (\%) or mean \pm SD.

Table 3. Cox Proportional-Hazards Model of MAHE and All-Cause Death ${ }^{\mathrm{a}}$

\begin{tabular}{|c|c|c|c|c|c|c|c|c|}
\hline & \multicolumn{4}{|c|}{ MAHE } & \multicolumn{4}{|c|}{ All-Cause Death } \\
\hline & HR & 95\% CI & PValue & P Value for Trend & HR & 95\% CI & PValue & P Value for Trend \\
\hline \multicolumn{9}{|c|}{ Unadjusted model } \\
\hline Low TNF- $\alpha$ & Reference & - & - & 0.0009 & Reference & - & - & 0.0002 \\
\hline Intermediate TNF- $\alpha$ & 1.7 & $1.23-1.98$ & 0.0007 & & 2.02 & $1.71-2.82$ & 0.0005 & \\
\hline High TNF- $\alpha$ & 2.26 & $1.81-2.73$ & 0.0003 & & 3.13 & $2.51-4.18$ & 0.0009 & \\
\hline \multicolumn{9}{|c|}{ Adjusted model } \\
\hline Low TNF- $\alpha$ & Reference & - & - & 0.0007 & Reference & - & - & 0.0008 \\
\hline Intermediate TNF- $\alpha$ & 1.32 & $1.02-1.68$ & 0.0009 & & 1.72 & $1.34-2.25$ & 0.0057 & \\
\hline High TNF- $\alpha$ & 1.74 & $1.05-1.93$ & 0.0006 & & 2.63 & $1.89-3.35$ & 0.0005 & \\
\hline
\end{tabular}

Abbreviations: CI, Confidence Interval; HR, Hazard Ratio; MAHE, Major Adverse Hepatic Events.

${ }^{a}$ Adjusted-for variables were age, BMI, diabetes. The covariates were added to this model only if identified statistically as predictors of MAHE and all-cause death (P < 0.05 )

ment of fatty liver and subsequently NASH $(20,21)$. Pentoxifylline, a phosphodiesterase inhibitor and non-specific TNF- $\alpha$ pathway antagonist, has been shown to improve the histological features of NASH in adult patients (22-24).
However, such results are not consistent in humans (22), which can be attributed to the diversity of different study populations or the lack of adjustment for several factors that might influence the results (23-25). 
A

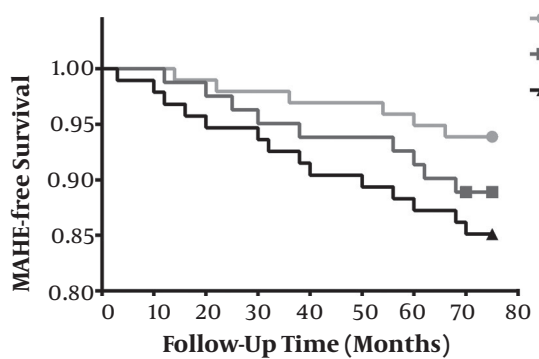

B

- Low TNF- $\alpha$

- Intermediate TNF- $\alpha$

- High TNF- $\alpha$

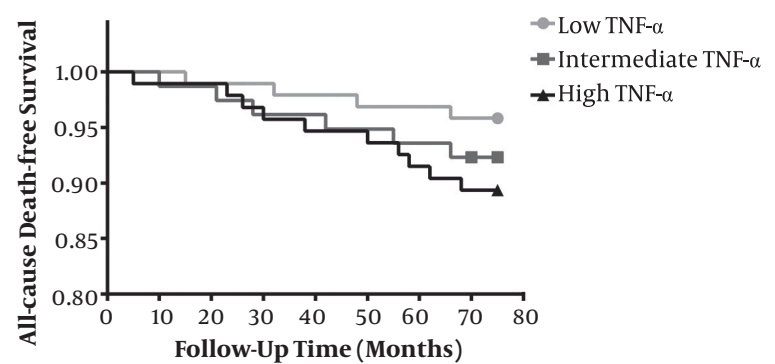

Figure 3. Major adverse hepatic events A, and all-cause death B, free survival curves for participants stratified by the concentration of TNF- $\alpha$

The role of TNF- $\alpha$ as a marker for NAFLD has been investigated in several cross-sectional analyses; however, the results show some contradictory (23-25). Therefore, multicenter longitudinal cohort studies are needed to demonstrate the relationship between TNF- $\alpha$ and NAFLD in the different population $(26,27)$. However, for the first time, the association of TNF- $\alpha$ with NAFLD in Chinese is demonstrated in this study, and will be a helpful supplement to TNF- $\alpha$ and NAFLD research. Relatively small and only onecenter participants are enrolled; further multi-center investigations on a large scale of participants are needed to confirm the present results.

PDFF measurement is an advanced MRI-based method for quantitatively assessing steatosis $(28,29)$. Compared with conventional MR imaging techniques, PDFF is a more objective, quantitative, and non-invasive imaging biomarker of liver fat content and the measurement is independent of scanner manufacturer, field strength, and other factors that may need to be adjusted to get the consistent results among different platforms. Thus, the results are more reproducible and valuable. Our analysis also testified that PDFF is an effective tool to evaluate liver fat fraction changes in NAFLD.

In summary, high TNF- $\alpha$ is found to be independent predictors of the risk of MAHE high serum. TNF- $\alpha$ level is strongly associated with increased incidences of MAHE and all-cause death in participants without NAFLD history who underwent MRI assessment.

\section{Acknowledgments}

Not applicable.

\section{Footnotes}

Authors' Contribution: Zhi Zhu and Shuofeng Li, data collection and analysis; Shuofeng Li, study design and manuscript writing. All authors read and approved the final submission.

Competing Interests: The authors have declared that no conflicts of interest exist.

\section{Funding/Support: None.}

Role of the Sponsor: The funding organizations are public institutions and had no role in the design and conduct of the study collection, management, and analysis of the data, or the preparation, review, and approval of the manuscript.

\section{References}

1. Chalasani N, Younossi Z, Lavine JE, Diehl AM, Brunt EM, Cusi K, et al. The diagnosis and management of non-alcoholic fatty liver disease: practice guideline by the American Gastroenterological Association, American Association for the Study of Liver Diseases, and American College of Gastroenterology. Gastroenterology. 2012;142(7):1592-609. doi: 10.1053/j.gastro.2012.04.001. [PubMed: 22656328].

2. Effendi K, Harada K, Hashimoto E, Kage M, Koike K, Kondo F. Pathological findings of NASH and NAFLD: for guidebook of NASH and NAFLD, 2015: The Japan society of hepatology. Hepatol Res. 2017:3-10.

3. Fattahi MR, Niknam R, Safarpour A, Sepehrimanesh M, Lotfi M. The Prevalence of Metabolic Syndrome In Non-alcoholic Fatty Liver Disease; A Population-Based Study. Middle East J Dig Dis. 2016;8(2):1317. doi: 10.15171/mejdd.2016.18. [PubMed: 27252820]. [PubMed Central: PMC4885612].

4. Tsochatzis EA, Bosch J, Burroughs AK. New therapeutic paradigm for patients with cirrhosis. Hepatology. 2012;56(5):1983-92. doi: 10.1002/hep.25915. [PubMed: 22729954].

5. Pais R, Ratziu V. Epidemiology and natural history of nonalcoholic fatty liver disease. J Clin Exp Hepatol. 2015;20(3):221-35.

6. Angulo P. Nonalcoholic fatty liver disease. 2017. p. 565-76.

7. Permutt Z, Le TA, Peterson MR, Seki E, Brenner DA, Sirlin C, et al. Correlation between liver histology and novel magnetic resonance imaging in adult patients with non-alcoholic fatty liver disease MRI accurately quantifies hepatic steatosis in NAFLD. Aliment Pharmacol Ther. 2012;36(1):22-9. doi: 10.1111/j.1365-2036.2012.05121.x. [PubMed: 22554256]. [PubMed Central: PMC3437221].

8. Lapadat AM, Jianu IR, Ungureanu BS, Florescu LM, Gheonea DI, Sovaila $S$, et al. Non-invasive imaging techniques in assessing non-alcoholic fatty liver disease: a current status of available methods. J Med Life. 2017;10(1):19-26. [PubMed: 28255371]. [PubMed Central: PMC5304366]. 
9. Nasr P, Forsgren MF, Ignatova S, Dahlstrom N, Cedersund G, Leinhard OD, et al. Using a 3\% Proton Density Fat Fraction as a Cut-Off Value Increases Sensitivity of Detection of Hepatic Steatosis, Based on Results From Histopathology Analysis. Gastroenterology. 2017;153(1):53-55 e7. doi: 10.1053/j.gastro.2017.03.005. [PubMed: 28286210].

10. European Association for Study of L, Asociacion Latinoamericana para el Estudio del H. EASL-ALEH Clinical Practice Guidelines: Noninvasive tests for evaluation of liver disease severity and prognosis. J Hepatol. 2015;63(1):237-64. doi: 10.1016/j.jhep.2015.04.006. [PubMed: 25911335].

11. Castera L. Invasive and non-invasive methods for the assessment of fibrosis and disease progression in chronic liver disease. Best Pract Res Clin Gastroenterol. 2011;25(2):291-303. doi: 10.1016/j.bpg.2011.02.003. [PubMed: 21497746].

12. Changchien C, Mwangi I, Yokoo T, Schroeder ME, Bydder M, Hamilton G. Comparison of Rates of Change in Segmental Liver Fat Using MRIDetermined Proton Density Fat Fraction. Radiological Society of North America 2010 Scientific Assembly and Meeting. 2010.

13. Yang YM, Seki E. TNFalpha in liver fibrosis. Curr Pathobiol Rep. 2015;3(4):253-61. doi: 10.1007/s40139-015-0093-z. [PubMed: 26726307]. [PubMed Central: PMC4693602].

14. Koek GH. [Treatment of non-alcoholic fatty liver disease]. Ned Tijdschr Geneeskd. 2011;155. A3181. [PubMed: 21649947].

15. Suissa S, Ernst P, Hudson M, Bitton A, Kezouh A. Newer diseasemodifying antirheumatic drugs and the risk of serious hepatic adverse events in patients with rheumatoid arthritis. Am J Med. 2004;117(2):87-92. doi: 10.1016/j.amjmed.2004.02.032. [PubMed: 15234643].

16. Zhao L, Wang X, Yang Y. Association between interleukin- 6 and the risk of cardiac events measured by coronary computed tomography angiography. Int J Cardiovasc Imaging. 2017;33(8):1237-44. doi: 10.1007/s10554-017-1098-y. [PubMed: 28233119].

17. Hedderich DM, Hasenberg T, Haneder S, Schoenberg SO, Kucukoglu O, Canbay A, et al. Effects of Bariatric Surgery on Non-alcoholic Fatty Liver Disease: Magnetic Resonance Imaging Is an Effective, Non-invasive Method to Evaluate Changes in the Liver Fat Fraction. Obes Surg. 2017;27(7):1755-62. doi: 10.1007/s11695-016-2531-3. [PubMed: 28064372].

18. McCullough AJ. The clinical features, diagnosis and natural history of nonalcoholic fatty liver disease. Clin Liver Dis. 2004;8(3):521-33. viii. doi: 10.1016/j.cld.2004.04.004. [PubMed: 15331061].

19. Kumar A, Singh AK, Panda PK, Nischal N, Soneja M. Non-alcoholic fatty liver disease diagnosis, grading and staging; a simplified tool for clinicians. J Adv Med. 2017;6(1):15. doi: 10.5958/2319-4324.2017.00003.7.

20. Metrakos P, Nilsson T. Non-alcoholic fatty liver disease-a chronic disease of the 21(st) century. J Biomed Res. 2017. doi: 10.7555/JBR.31.20160153. [PubMed: 28550272].

21. Hammerich L, Tacke F. Interleukins in chronic liver disease: lessons learned from experimental mouse models. Clin Exp Gastroenterol. 2014;7:297-306. doi: 10.2147/CEG.S43737. [PubMed: 25214799]. [PubMed Central: PMC4158890].

22. Wellen KE, Hotamisligil GS. Obesity-induced inflammatory changes in adipose tissue. JClin Invest. 2003;112(12):1785-8. doi:10.1172/JCI20514. [PubMed: 14679172]. [PubMed Central: PMC297006].

23. Abiru S, Migita K, Maeda Y, Daikoku M, Ito M, Ohata K, et al. Serum cytokine and soluble cytokine receptor levels in patients with nonalcoholic steatohepatitis. Liver Int. 2006;26(1):39-45. doi: 10.1111/j.14783231.2005.01191.x. [PubMed: 16420507].

24. Hui JM, Hodge A, Farrell GC, Kench JG, Kriketos A, George J. Beyond insulin resistance in NASH: TNF-alpha or adiponectin? Hepatology. 2004;40(1):46-54. doi: 10.1002/hep.20280. [PubMed:15239085].

25. Musso G, Gambino R, Durazzo M, Biroli G, Carello M, Faga E, et al. Adipokines in NASH: postprandial lipid metabolism as a link between adiponectin and liver disease. Hepatology. 2005;42(5):1175-83. doi: 10.1002/hep.20896. [PubMed:16231364].

26. Zahran WE, Salah El-Dien KA, Kamel PG, El-Sawaby AS. Efficacy of Tumor Necrosis Factor and Interleukin-10 Analysis in the Follow-up of Nonalcoholic Fatty Liver Disease Progression. Indian J Clin Biochem. 2013;28(2):141-6. doi: 10.1007/s12291-012-0236-5. [PubMed: 24426199]. [PubMed Central: PMC3613495].

27. Seo YY, Cho YK, Bae JC, Seo MH, Park SE, Rhee EJ, et al. Tumor Necrosis Factor-alpha as a Predictor for the Development of Nonalcoholic Fatty Liver Disease: A 4-Year Follow-Up Study. Endocrinol Metab (Seoul). 2013;28(1):41-5. doi: 10.3803/EnM.2013.28.1.41. [PubMed: 24396649]. [PubMed Central: PMC3811803]

28. Idilman IS, Aniktar H, Idilman R, Kabacam G, Savas B, Elhan A, et al. Hepatic steatosis: quantification by proton density fat fraction with MR imaging versus liver biopsy. Radiology. 2013;267(3):767-75. doi: 10.1148/radiol.13121360. [PubMed: 23382293].

29. Tang A, Desai A, Hamilton G, Wolfson T, Gamst A, Lam J, et al. Accuracy of MR imaging-estimated proton density fat fraction for classification of dichotomized histologic steatosis grades in nonalcoholic fatty liver disease. Radiology. 2015;274(2):416-25. doi: 10.1148/radiol.14140754. [PubMed: 25247408]. [PubMed Central: PMC4314291] 